\title{
Single pulse profiles and the Lorentz factor of emitting particles in pulsar magnetospheres
}

\author{
N. Rathnasree \\ Raman Research Institute, Bangalore, 560080, INDIA
}

\section{Introduction}

If each significant component in a single pulse from a radio pulsar represents emission from a single site and the intensity at longitudes other than the peak arises from the forward beaming of this emission, the angular distribution of intensity within a component is given by (Zheleznyakov, \& Shaposhnikov 1972),

$$
I_{\nu}(\phi)=K \frac{\left(1-\beta^{2}\right)^{(2-\alpha)}}{\left(1-\beta \cos \left(\phi-\phi_{0}\right)\right)^{\frac{(3-\alpha)}{2}}} \nu^{\alpha}
$$

Here, $\beta=\frac{v}{c}$, with $v$ the velocity of the emitting particles at the emission site. $\alpha$ is the spectral index of emission in the rest frame of the emitting particles, and is also the spectral index as seen by the observer. $\nu$ is the observed frequency of the radiation. $K \nu^{\alpha}$ is the intensity in the rest frame of the emitting particles. $\phi_{0}$ is the angular co-ordinate corresponding to the direction of motion of the emitting particles, and $\phi$ is the angular co-ordinate at different regions in a single component.

\section{Lorentz Factor distribution}

Remarkably good fits (Fig. 1a) to the single pulse profiles are obtained to the above equation, indicating that the underlying assumption of components arising from beamed emission is good. A distribution of $\gamma$ for emitting particles can thus be obtained from this procedure by equating the inverse of the widths of subpulses to the Lorentz factor of emitting particles. This distribution when observed for components in a few thousand pulses is seen to have stable characteristics. For $1929+10$, at $1414 \mathrm{MHz}$, this is shown in Fig. 1b, where the number of occurences of components with a value of $\gamma$ in a given range is plotted as a histogram. It is remarkable that a stable distribution of the form $N(\gamma) \approx \gamma^{-w}$, at the high $\gamma$ end, is obtained, for all the pulsars studied. Furthermore, $w \approx-2.5--2.8$ in all these cases. Below the low $\gamma$ turnover, a fit of the form $N(\gamma) \approx e^{-\left(\gamma_{c} / \gamma\right)}$ seems consistent with the data. Most calculations of pair production cascades in pulsar magnetospheres (Arons 1981, Daugherty \& Harding 1982, Beskin et al 1988) predict particle energy distribution in the pulsar magnetosphere similar to what is seen for the present data. 
Our suggestion is based on the Rutherman and Sutherland (1975) belief, that the polar gap discharges through a group of localized sparks, spaced at the distance nearly equals to the height of the polar gap. The fixed position of the sparks, which one needs to achieve a stable structure of an integrated profile, may be conditioned by pinning the sparks to irregularities in the magnetic field.

Based on Ruderman and Sutherland (1975) belief that the space between the sparks $\Delta L$ is nearly equals to the height $h$ of the polar gap, we evaluate the value of $h$

$$
h=\Delta L=R_{p} / n=R(2 \pi R / c P)^{2} / n .
$$

Here $R_{p}$ is the radius of polar cap region, $n$ is the number of components, $R$ is the radius of a star and $P$ is a period of pulsar. For $R=10^{6} \mathrm{~cm}$ and $n$ $=6$, we obtain $h=3 \times 10^{3} \mathrm{~cm}$, which is in good accordance with a theoretical estimation $h=5 \times 10^{3} \mathrm{~cm}$ (Ruderman and Sutherland 1975).

\section{Conclusion}

We suggest that the emission region inside of the cone of the open magnetic field lines represent a mosaic bunch of the discrete outflows of relativistic charges along magnetic field lines, injected by mosaic group of localized sparks in the polar cap.

We suggest that mode changing may be produced by the change of an activity of some sparks pattern without rearranging the structure of the emission region.

Acknowledgments. Authors are grateful to A.Lyne and F.G.Smith for providing the data of observations at Jodrell Bank observatory, R.Wielebinski, A.Jessner, W.Sieber, J.Seierdakis, K.Xiluori, J.Gil, V.Malofeev and I.Malov for assistance in observations at Effelsberg observatory. This work was supported partly by International Science Foundation (Grant RN-6300) and European Southern observatory (Grant A-04-083).

\section{References}

Foster R.S., Fairhead L., Backer D.C., 1991, ApJ, 387, 687.

Hesse K.H., 1973, A\&A, 27, 373

Oster L., Sieber W.,1977, ApJ, 58, 303.

Rankin J.M., 1983, ApJ, 274, 333.

Ruderman M.A., Sutherland P.C., 1975, ApJ, 196, 51.

Wu Xinji, Xu Wenand, Rankin J.M., 1992, in Proc, IAU Symp. N 128, Pedagogical Univ. Press, p.172-175 

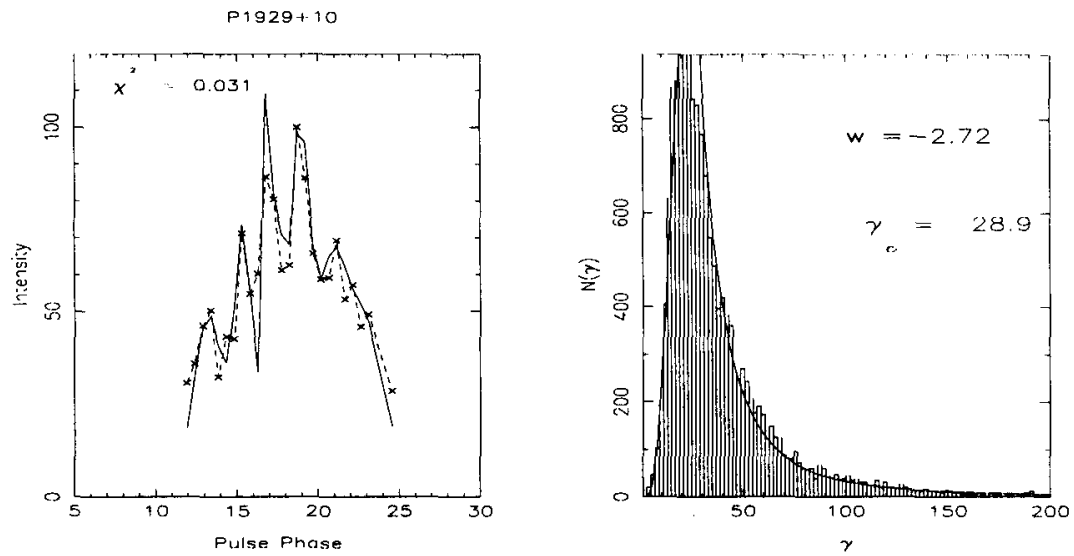

Figure 1. (a) Data (crosses joined with broken line) from a single pulse fitted (solid line) with beaming pattern obtained from Eq. (1) for a discrete number of emission sites. (b) Distribution of particle energies $\gamma$ determined as discussed in text. The number of data points (sub-pulse peaks) corresponding to $\gamma$ within a given range are plotted as a histogram.

\section{Conclusions}

In conclusion, it is shown that components in single pulses arise from the relativistic beaming of emission from discrete emission sites. The observation that different components in the average profile do not have markedly different distributions in $\gamma$, has important implications for theories of emission for these components. The overall distribution of particle energies giving rise to this emission is seen to be having a characteristic shape which is seen to be very similar for all the pulsars studied. The study of the energy spectrum of emitting particles offers the first close contact between the physical processes responsible for radio emission in pulsar magnetospheres and the observations.

Acknowledgments. The data used in this study was obtained in collaboration with Joanna M. Rankin, Amy Carlow, Vera Izvekova and Sveta Suleymanova. A pleasure to thank Avinash Deshpande, V. Radhakrishnan, Patrick Das Gupta, C.S. Shukre, Joanna Rankin and R. Ramachandran for many useful discussions and suggestions. Arecibo Observatory is operated by Cornell University under contract to the National Science Foundation.

\section{References}

Arons, J., 1981, in Pulsars, IAU Sumposium 95, (eds.) W. Seiber \& R. Wielebenski, (D. Reidel).

Beskin, V.S., Gurevich, A.V. \& Istomin, Ya. N., 1988 Ap\&SS, 146, 205.

Daugherty, J.K. \& Harding, A.K., 1982, ApJ, 273, 337.

Zheleznyakov, V.V. \& Shaposhnikov, V.E., 1972, Ap\&SS, 18, 141. 